\title{
Sleep disturbances and cognitive decline: recommendations on clinical assessment and the management
}

\author{
B. GUARNIERI', G. CERRONI', S. SORBI² \\ ${ }^{1}$ Center of Sleep Medicine, Villa Serena Hospital, Città S. Angelo, Pescara, Italy; ${ }^{2}$ Department of \\ Neuroscience, Psychology, Drug Research and Child Health (NEUROFARBA), University of Florence, \\ Florence, Italy
}

\begin{abstract}
A B S T R A C T
In 2004, in Genoa (Italy), the Italian Dementia Research Association (SINDem) was born. The first congress of this new scientific society took place in Rome in 2006.

SINDem soon recognized the importance to investigate sleep problems in cognitive decline and created a national "sleep study group "composed by neurologists and sleep specialists.

In 2012, The SINDem study group, in close relationship with the Italian Association of sleep medicine (AIMS), published the study "Prevalence of sleep disturbances in mild cognitive impairment and dementing disorders: a multicenter Italian clinical cross-sectional study on 431 patients ", confirming the high prevalence of sleep disturbances in a wide Italian population of persons with cognitive decline. The study was supported by a grant from the Italian Minister of Health and was conducted with the fundamental contribution of the Italian National Research Center (CNR).

In 2014, the same group published the paper "Recommendations of the Sleep Study Group of the Italian Dementia Research Association (SINDem) on clinical assessment and management of sleep disorders in individuals with mild cognitive impairment and dementia: a clinical review". The recommendations are wide and directed to professionals (neurologists but not exclusively) to try to establish uniform levels of care, promote collaborative studies into areas of uncertainty, and define the qualitative characteristics of Dementia Reference Centers about sleep disturbances.
\end{abstract}

Key words

Sleep-wake rhythm $\bullet$ Sleep disturbances $\bullet$ Dementias

\section{Introduction}

The prevalence of sleep disorders increases with age suggesting that sleep is impaired by aging itself and/or by aging-related conditions as neurodegenerative diseases (Ancoli-Israel and Ayalon, 2006; Stepnowsky and Ancoli-Israel, 2008; Yaffe et al., 2014).

The high prevalence of sleep disorders reported in neurodegenerative diseases and dementia in particular, could be explained by intrinsic changes specific to each form of dementia but also by the high frequency of other less specific conditions potentially affect- ing sleep in these patients (chronic pain, depression, prolonged bedrest and medication use) (Vitiello and Borson, 2001;Anderson and Bradley, 2013).

The diagnosis and management of sleep disturbances in patients with cognitive decline has wide clinical and social implications. Poor sleep and rest-activity rhythm disruption result in an increased risk of morbidities and mortality in demented patients and cause caregivers exhaustion often leading to the institutionalization of patients (McCurry et al., 2007).

However, dementing disorders are not only present in older adults and even if sleep disturbances are consis- 
tently reported across moderate to severe $\mathrm{AD}$ patients, they often precede by decades the clinical presentation of dementia so the relationship between sleep and cognitive decline is evolving towards new directions.

Circadian sleep rhythm disturbances (CSRD) affect as many as a quarter of Alzheimer's (AD) disease patients during some stage of their illness. Circadian control of the sleep-wake cycle is mediated by the suprachiasmatic nuclei ( $\mathrm{SCN})$ of the hypothalamus and, at a lesser degree, by subcortical structures such as the basal forebrain, dorsal and central superior raphe nuclei, and the reticular formation of the pons and medulla which seem to be involved in the initiation of sleep and the oscillation between REM and non-REM states. The circadian influences of SCN neurons are distributed through different target organs by neural and humoral signals particularly circulating melatonin. The nucleus controls melatonin secretion via a multisynaptic pathway determining changes in the biological clock, core body temperature, and sleep.

All of these structures potentially could be damaged because of $\mathrm{AD}$, frontotemporal dementia (FTD and possibly other kinds of degenerative dementia bringing to many sleep architecture and continuity changes seen in these illnesses (Harper et al., 2001; Weldemichael and Grossberg, 2010; Volicer et al., 2012).

On the other hand, sleep and sleep - wake disturbances "per se" seem to be risk factors for dementia (Tranah et al., 2011; Lim et al., 2013) and can precede dementia symptoms by several years. In Lewy body dementia (LBD) and Parkinson disease dementia (PDD) sleep disorders with daytime hypersomnolence and nocturnal parasomnias, REM behavior disorder (RBD) in particular, have been recognized to be prodromal features and they may contribute to the typical fluctuations of the disease (Boeve et al., 2001).

In recent years, several studies have suggested that sleep may influence the pathogenesis of $A D$ and other dementias. The knowledge of the molecular and cellular basis of this relationship is evolving and we find scientific editorials entitled as follows: "Don't lose sleep over neurodegeneration: it helps clear amyloid beta" (Malkki, 2013).

Alterations in the sleep-wake cycle seem to be related to degeneration in melanopsin-expressing retinal ganglion cells: visual and retinal abnormalities as early manifestation of retinal amyloid beta $(\mathrm{A} \beta)$ plaque pathology have been described in $\mathrm{AD}$ patients, animal models and in preclinical AD ( $\mathrm{La}$ Morgia et al., 2011; Koronyo et al., 2012).

In addition, the genetic control of sleep is involved in cognitive decline and a larger evidence is expected (Wu et al., 2006; Tseng et al., 2010).

Hypothalamic polypeptides such as melanin-concentrating hormone (MCH) and HCRT-1(syn orexin-A) promote sleep-wake regulation, energy homeostasis and seem to impact on cognitive functions (Slats et al., 2012; Fronczek et al., 2012).

In both humans and mouse models, $\mathrm{A} \beta$ increases during wakefulness and decreases during sleep: sleep problems may increase soluble $A \beta$ levels over time, leading to an increased possibility of amyloid plaque deposition with further sleep disruption and, subsequently, symptomatic AD.

In mouse models of $\mathrm{AD}$, chronic sleep deprivation augmented amyloid plaque formation, while increasing sleep with an orexin receptor antagonist reduced amyloid plaques (Dobrowolska et al., 2014; Lucey and Bateman, 2014; Spira et al., 2013)

Moreover, animal studies demonstrated that the cerebral interstitial space increases by more than $60 \%$ during sleep, resulting in efficient convective clearance of $\mathrm{A} \beta$ and other toxic compounds (Xie et al., 2013).

A good sleep consolidation seems to attenuate the effect of APOE genotype on incident AD and development of neurofibrillary tangle pathology: interventions to obtain a better sleep consolidation should be relevant to reduce the risk of AD in APOE ع4+ individuals (Lim et al., 2013).

An association between sleep-disordered breathing (SDB) and cerebrospinal fluid $\mathrm{AD}$ biomarkers in cognitively normal elderly individuals has been demonstrated and, consequently, therapies for SDB such as continuous positive airway pressure (CPap) ventilations could delay the onset of MCI or dementia in normal elderly individuals (Osorio et al., 2014).

Even in dementia patients, the failure to recognize clinically relevant obstructive sleep apnea syndrome (OSAS) is important as the disease is associated with significant morbidity and mortality and OSAS is an independent risk factor for the development of cardiovascular diseases and stroke. Consequently, to detect sleep disordered breathing is particularly relevant in vascular dementia (VaD) (Wallace et al., 2012; Parati et al., 2012; Hermann and Bassetti, 2009).

The use of CPap has been shown to decrease sleep disturbances in AD patients with OSAS and some 
studies have shown positive improvement in neuropsychological functioning (Cooke et al., 2009). In 2011, the Alzheimer's Association and the National Institute on Aging (NIA) of the National Institutes of Health (NIH) published new diagnostic guidelines for $\mathrm{AD}$, expanding the definition of $\mathrm{AD}$ to include two new phases of the illness: (1) presymptomatic and (2) mildly symptomatic but pre-dementia, along with (3) dementia caused by $\mathrm{AD}$. This reflects current thinking that $\mathrm{AD}$ begins decades before memory and thinking symptoms are detectable: this new classification is aimed to find distinct and measurable markers in the brains of affected people early in the natural history of the illness (McKhann et al., 2011).

As described, several sleep studies focused on preclinical stages of dementing illnesses trying to use sleep and its pathophysiology to gain modifiable processes possibly delaying the beginning and the progression of the disease.

\section{Recommendations on clinical assessment and themanagementofsleepdisturbances in patients with cognitive decline}

In 2004, in Genoa, Italy, a group of neurologists founded the Italian Dementia Research Association (SINDem). The first congress of this new society took place in Rome in 2006: Professor Carlo Caltagirone (Rome, Italy) and Professor Sandro Sorbi (Florence, Italy) were nominated first president and elected president, respectively.

SINDem soon recognized the importance to investigate sleep problems in cognitive decline and created a national "sleep study group": the neurologists and sleep specialists involved, organized a wide multicenter study on the prevalence of sleep disturbances in mild cognitive impairment (MCI) and dementia patients. The work was supported by a grant from the Italian Minister of Health and by the Italian National Research Center (CNR): 431 consecutive community dwelling patients with dementia or MCI were enrolled by 10 Italian neurological centers over a period of 6 months. Insomnia, hypersomnolence- excessive daytime sleepiness (EDS), SDB, RBD restless legs syndrome (RLS) were investigated using questionnaires and scales with acceptable values of sensitivity and specificity. In that period, several tests were not com- pletely validated in dementia patients and in the Italian population, but widely used in sleep studies all over the world. Only several months after the end of the study, the Pittsburg sleep quality Index (PSQI) was validated in Italian persons with cognitive decline (Curcio et al., 2013) and a new Italian scale for sleep continuity was validated (Manni et al., 2013).

Moreover, the Mayo Clinic sleep questionnaire to screen RBD in aging and dementia cohort was validated after the beginning of the SINDem study and the Mayo core question for a clinical diagnosis of RBD, supported by the direct caregiver, substantially resembled the one used in the Italian study (Boeve et al. 2011).

The clinical cross sectional study was published in 2012 (Guarnieri et al., 2012) and confirmed the high prevalence of several sleep disturbances in MCI, $\mathrm{AD}, \mathrm{VaD}, \mathrm{FTD}, \mathrm{LBD}$ and PDD: almost $60 \%$ of the patients had symptoms clinically compatible with SDB and more than 50\% had EDS and insomnia. Clinically probable RBD was particularly frequent in about $25 \%$ of the patients, whereas RLS, the less frequent disorder, showed a prevalence of about $6 \%$ not dissimilar from that found in normal elderly populations. The score of depressive symptoms was more severe in subjects presenting any of the investigated sleep disorders.

Taking AD patients as the reference category, the presence of at least one of any of the investigated disturbances was two times more common in patients with $\mathrm{VaD}$ or LBD/PDD, but this difference was non statistically significant. Patients with MCI had a frequency of sleep disturbances of any type equal to that of patients with $\mathrm{AD}$. Insomnia frequency was identical in $\mathrm{AD}$ and FTD patients but was about 2.5 and 1.5 times more frequent in $\mathrm{VaD}$ and $\mathrm{LBD} / \mathrm{PDD}$ patients, respectively. SDB was about two times more frequent in patients with $\mathrm{VaD}$ than in those with $\mathrm{AD}$. RBD was 2.6 times more frequent in LBD/PDD patients, whereas no difference of occurrence was observed for RLS among the different types of dementia. EDS was more frequent among $\mathrm{VaD}$ and $\mathrm{LBD} / \mathrm{PDD}$ patients than among $\mathrm{AD}$ patients, but these differences were only of borderline statistical significance.

An important finding was that different sleep disturbances occurred almost invariably in association in the same patient, without evident specific patterns of association except for RBD and synucleinopathies, confirming the need to deeply investigate each kind of sleep disturbance to reach correct therapies, avoid- 
ing interferences among treatments for different sleep disturbances. Following the multicenter Italian prevalence study, the SINDem Sleep study group, in close relationship with the Italian Association of Sleep Medicine (AIMS) published recommendations on clinical assessment and the management of sleep disorders in individuals with MCI and dementia. The Italian Neurological Society (SIN) officially approved recommendations on February 2014 (Guarnieri et al., 2014). The recommendations are directed to professionals (neurologists but not exclusively) involved in the complex diagnostic workup of dementias: they are aimed to establish uniform levels of care, promote collaborative research into areas of uncertainty, and define the qualitative characteristics of Dementia Reference Center about sleep disturbances. They were prepared based on scientific evidence and they reflected the professional judgment and opinions of the members of the SINDem sleep study group only if such evidence was insufficient or contradictory. Evidence and recommendation levels were established for insomnia, EDS, CSRD, RBD, SDB end RLS.

Three important starting recommendations are the following

- "Sleep disorders are frequent and tend to occur almost invariably in association in patients with cognitive decline: they have to be always carefully investigated using an in-depth sleep history, a physical examination and questionnaires and scales, whenever possible validated with acceptable and definite values of sensitivity and specificity, directly filled by the patient with the support of the direct caregiver, when possible"

- "Recommendations for older adults can be considered a good instrument also for individuals with MCI and dementia of the same age when specific recommendations are unsatisfactory or insufficient"

- "Instrumental supports to investigate sleep problems should be considered in selected patients, after a referral to a sleep specialist"

Moreover, the authors suggest that hypersomnolence should be always addressed in patients with MCI, dementia, and in particular in nursing home residents.

Regarding treatments, non-pharmacologic strategies are recommended as the most appropriate starting treatment. Lifestyle factors have to be considered and the management of sleep disturbances should be categorized according to the presence of several sleep disorders, which can require different kinds of treatment.

Among recommendations for insomnia, we find that pharmacological therapy can be recommended for short-term treatments: any pharmacological therapy should be regularly

reviewed (almost every 4 weeks) and chronic use of hypnotic drugs should be addressed only

following selective and specific indications.

The paper suggests that, whenever possible, insomnia should be categorized in starting and maintaining insomnia, considering other sleep disturbances in comorbidity: in maintaining insomnia, in particular, the presence of SDB and/or parasomnias should be carefully investigated. To reduce or eliminate medications that may contribute to sleep apnea is recommended. In the presence of SDB, long-acting benzodiazepines should be avoided or used with caution: if they are strictly indicated, the dosage should be the lowest possible, in particular if SDB is not treated or cannot be treated for compliance problems.

Regarding EDS and CSRD, authors recommend multimodal approaches as combining bright light exposure during the day, decreasing light exposure at night, physical and social activity, regular schedules of wake and bedtime and melatonin administration.

In patients affected by RBD, except from medications, particular attention has been devoted to environmental safety to minimize injury and decrease the risk from falling off the bed: to avoid alcohol intake and/or withdrawal, the use of antidepressants, neuroleptics as well as beta-blockers, and tramadol, which can trigger or exacerbate RBD, have been widely considered for the management.

For the treatment of all kinds of SDB (OSA, Central sleep apnea, Cheyne Stokes breathing, etc.), patients should be referred to a sleep specialist and/or sleep center to define diagnosis and treatments. Even in patients with cognitive decline, CPap is recommended as the first-line of treatment independently of age and cognitive impairment: authors say, "Offering CPap treatment to patients who are able to comply is certainly reasonable" and the help from a family member or caregiver is generally necessary.

Considering that no extensive studies on RLS treatment in dementia patients have been performed to reach specific levels of recommendations, recom- 
mendations for general population and in particular for older individuals can be adopted in patients with cognitive decline.

SINDem recommendations on clinical assessment and the management of sleep problems in patients with cognitive decline can be considered a valid instrument to help patients and to further discuss and investigate the close relationships between sleep and dementia.

\section{Acknowledgments}

We thank Mario Guazzelli, a great scientist and a precious friend, for his exemplum in sleep research and clinical practice.

\section{References}

Ancoli-Israel S. and Ayalon L. Diagnosis and treatment of sleep disorders in older adults. Am. $J$. Geriatr. Psychiatry, 14: 95-103, 2006.

Anderson K.N. and Bradley AJ. Sleep disturbance in mental health problems and neurodegenerative disease. Nat. Sci. Sleep, 5: 61-75, 2013.

Boeve B.F., Molano J.R., Ferman T.J., Smith G.E., Lin S.C., Bieniek K., Haidar W., Tippmann-Peikert M., Knopman D.S., Graff-Radford N.R., Lucas J.A., Petersen R.C., Silber M.H.. Validation of the Mayo Sleep Questionnaire to screen for REM sleep behavior disorder in an aging and dementia cohort. Sleep Med., 12: 445-453, 2011.

Boeve B.F., Silber M.H., Ferman T.J., Lucas J.A., Parisi J.E. Association of REM sleep behavior disorder and neurodegenerative disease may reflect an underlying synucleinopathy. Mov. Disord., 16: 622-630, 2001.

Cooke J.R., Ayalon L., Palmer B.W., Loredo J.S., Corey-Bloom J., Natarajan L., Liu L., Ancoli-Israel S. Sustained use of CPAP slows deterioration of cognition, sleep, and mood in patients with Alzheimer's disease and obstructive sleep apnea: a preliminary study. J. Clin. Sleep Med., 5: 305-309, 2009.

Curcio G., Tempesta D., Scarlata S., Marzano C., Moroni F., Rossini P.M., Ferrara M., De Gennaro L. Validity of the Italian version of the Pittsburgh Sleep Quality Index (PSQI). Neurol. Sci., 34: 511$519,2013$.

Dobrowolska J.A., Kasten T., Huang Y., Benzinger T.L., Sigurdson W., Ovod V., Morris J.C., Bateman R.J. Diurnal patterns of soluble amyloid precursor protein metabolites in the human central nervous system. PLoS One, 9: e89998.2014.

Fronczek R., van Geest S., Frölich M., Overeem S., Roelandse F.W., Lammers G.J., Swaab D.F. Hypocretin (orexin) loss in Alzheimer's disease. Neurobiol. Aging, 33: 1642-1650, 2012.

Guarnieri B., Adorni F., Musicco M., Appollonio I., Bonanni E., Caffarra P., Caltagirone C., Cerroni G., Concari L., Cosentino F.I., Ferrara S., Fermi S., Ferri R., Gelosa G., Lombardi G., Mazzei D., Mearelli S., Morrone E., Murri L., Nobili F.M., Passero S., Perri R., Rocchi R., Sucapane P., Tognoni G., Zabberoni S., Sorbi S. Prevalence of sleep disturbances in mild cognitive impairment and dementing disorders: a multicenter Italian clinical cross-sectional study on 431 patients. Dement. Geriatr. Cogn. Disord., 33: 50-58, 2012.

Guarnieri B., Musicco M., Caffarra P., Adorni F., Appollonio I., Arnaldi D., Bartoli A., Bonanni E., Bonuccelli U., Caltagirone C., Cerroni G., Concari L., Cosentino F.I., Fermi S., Ferri R., Gelosa G., Lombardi G., Mearelli S., Nobili F., Passero S., Perri R., Rocchi R., Sucapane P., Tognoni G., Zabberoni S., Sorbi S. Recommendations of the Sleep Study Group of the Italian Dementia Research Association (SINDem) on clinical assessment and management of sleep disorders in individuals with mild cognitive impairment and dementia: a clinical review. Neurol. Sci., 35: 13291348, 2014.

Harper D.G., Stopa E.G., McKee A.C., Satlin A., Harlan P.C., Goldstein R., Volicer L. Differential circadian rhythm disturbances in men with Alzheimer disease and frontotemporal degeneration. Arch. Gen. Psychiatry., 58: 353-360, 2001.

Hermann D.M. and Bassetti C.L. Sleep-related breathing and sleep-wake disturbances in ischemic stroke. Neurology, 73: 1313-1322, 2009.

Koronyo Y., Salumbides B.C., Black K.L., KoronyoHamaoui M. Alzheimer's disease in the retina: imaging retinal $a \beta$ plaques for early diagnosis and therapy assessment. Neurodegener. Dis., 10: 285-293, 2012.

La Morgia C., Ross-Cisneros F.N., Hannibal J., Montagna P., Sadun A.A., Carelli V. Melanopsinexpressing retinal ganglion cells: implications for human diseases. Vision Res., 51: 296-302, 2011.

Lim A.S., Kowgier M., Yu L., Buchman A.S., Bennett D.A. Sleep Fragmentation and the Risk of Incident Alzheimer's Disease and Cognitive Decline in Older Persons. Sleep, 36: 1027-1032, 2013.

Lim A.S., Yu L., Kowgier M., Schneider J.A., Buchman A.S., Bennett D.A. Modification of the relation- 
ship of the apolipoprotein E $\varepsilon 4$ allele to the risk of Alzheimer disease and neurofibrillary tangle density by sleep. JAMA Neurol., 70: 1544-1551, 2013.

Lucey B.P. and Bateman R.J. Amyloid- $\beta$ diurnal pattern: possible role of sleep in Alzheimer's disease pathogenesis. Neurobiol. Aging., 35: S29-34, 2014.

Malkki H. Alzheimer disease: Sleep alleviates AD-related neuropathological processes. Nat. Rev. Neurol., 9: 657, 2013.

Manni R., Sinforiani E., Zucchella C., Terzaghi M., Rezzani C. A sleep continuity scale in Alzheimer's disease: validation and relationship with cognitive and functional deterioration. Neurol Sci., 34: 701705, 2013.

McCurry S.M., Logsdon R.G., Teri L., Vitiello M.V. Sleep disturbances in caregivers of persons with dementia: contributing factors and treatment implications. Sleep Med Rev., 11: 143-153, 2007.

McKhann G.M., Knopman D.S., Chertkow H., Hyman B.T., Jack C.R. Jr, Kawas C.H., Klunk W.E., Koroshetz W.J., Manly J.J., Mayeux R., Mohs R.C., Morris J.C., Rossor M.N., Scheltens P., Carrillo M.C., Thies B., Weintraub S., Phelps C.H. The diagnosis of dementia due to Alzheimer's disease: recommendations from the National Institute on Aging-Alzheimer's Association workgroups on diagnostic guidelines for Alzheimer's disease. Alzheimers Dement., 7: 263-269, 2011.

Osorio R.S., Ayappa I., Mantua J., Gumb T., Varga A., Mooney A.M., Burschtin O.E., Taxin Z., During E., Spector N., Biagioni M., Pirraglia E., Lau H., Zetterberg H., Blennow K., Lu S.E., Mosconi L., Glodzik L., Rapoport D.M., de Leon M.J. The interaction between sleep-disordered breathing and apolipoprotein E genotype on cerebrospinal fluid biomarkers for Alzheimer's disease in cognitively normal elderly individuals. Neurobiol. Aging, 35: 1318-1324, 2014.

Parati G., Lombardi C., Hedner J., Bonsignore M.R., Grote L., Tkacova R., Levy P., Riha R., Bassetti C., Narkiewicz K., Mancia G., McNicholas W.T., European Respiratory Society, EU COST ACTION B26 members. Position paper on the management of patients with obstructive sleep apnea and hypertension: joint recommendations by the European Society of Hypertension, by the European Respiratory Society and by the members of European COST (Cooperation in Scientific and Technological research) ACTION B26 on obstructive sleep apnea. J. Hypertens., 30: 633-646, 2012.

Slats D., Claassen J.A., Lammers G.J., Melis R.J., Verbeek M.M., Overeem S. Association between hypocretin- 1 and amyloid- $\beta 42$ cerebrospinal fluid levels in Alzheimer's disease and healthy controls. Curr. Alzheimer Res., 9: 1119-1125, 2012.

Spira A.P., Gamaldo A.A., An Y., Wu M.N., Simonsick E.M., Bilgel M., Zhou Y., Wong D.F., Ferrucci L., Resnick S.M. Self-reported sleep and $\beta$-amyloid deposition in community-dwelling older adults. JAMA Neurol., 70: 1537-1543, 2013.

Stepnowsky C.J. and Ancoli-Israel S. Sleep and Its Disorders in Seniors. Sleep Med. Clin, 3: 281-293, 2008.

Tranah G.J., Blackwell T., Stone K.L., Ancoli-Israel S., Paudel M.L., Ensrud K.E., Cauley J.A., Redline S., Hillier T.A., Cummings S.R., Yaffe K., SOF Research Group. Circadian activity rhythms and risk of incident dementia and mild cognitive impairment in older women. Ann. Neurol., 70: 722-732, 2011.

Tseng I.J., Liu H.C., Yuan R.Y., Sheu J.J., Yu J.M., $\mathrm{Hu}$ C.J. Expression of inducible nitric oxide synthase (iNOS) and period 1 (PER1) clock gene products in different sleep stages of patients with cognitive impairment. J. Clin. Neurosci., 17: 11401143, 2010.

Vitiello M.V. and Borson S. Sleep disturbances in patients with Alzheimer's disease: epidemiology, pathophysiology and treatment. CNS Drugs., 15: 777-796, 2001.

Volicer L., Harper D.G., Stopa E.G. Severe impairment of circadian rhythm in Alzheimer's disease. J. Nutr. Health Aging, 16: 888-890, 2012.

Wallace D.M., Ramos A.R., Rundek T. Sleep disorders and stroke. Int. J. Stroke, 7: 231-242, 2012.

Weldemichael D.A. and Grossberg G.T. Circadian rhythm disturbances in patients with Alzheimer's disease: a review. Int. J. Alzheimers Dis., 2010: 716453-716462, 2010.

Wu Y.H., Fischer D.F., Kalsbeek A., Garidou-Boof M.L., van der Vliet J., van Heijningen C., Liu R.Y., Zhou J.N., Swaab D.F. Pineal clock gene oscillation is disturbed in Alzheimer's disease, due to functional disconnection from the "master clock". FASEB J., 20: 1874-1876, 2006.

Xie L., Kang H., Xu Q., Chen M.J., Liao Y., Thiyagarajan M., O'Donnell J., Christensen D.J., Nicholson C., Iliff J.J., Takano T., Deane R., Nedergaard M. Sleep drives metabolite clearance from the adult brain. Science, 342: 373-377, 2013.

Yaffe K., Falvey C.M., Hoang T. Connections between sleep and cognition in older adults. Lancet Neurol., 13: 1017-1028, 2014. 